\title{
REPORTAJES ESPECIALES EN LOS CIBERMEDIOS. ANÁLISIS DE DIEZ AÑOS DE PREMIOS GOYA Y OSCAR AWARDS EN ELPAIS.COM (2005-2014)
}

\author{
Special features in online journalism. Ten years of \\ Premios Goya and Oscar Awards \\ in Elpais.com (2005-2014)
}

Pere Freixa

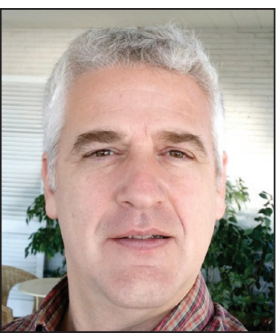

Pere Freixa es licenciado y doctor en bellas artes por la Universitat de Barcelona y especialista en multimedia interactivo por la Universitat Politécnica de Catalunya. Es profesor de la Facultad de Comunicación de la Universitat Pompeu Fabra desde su fundación. En 2009 se incorporó al grupo de investigación DigiDoc como especialista en imagen fotográfica, interfaz gráfica e interacción. http://orcid.org/0000-0002-9199-1270

Universitat Pompeu Fabra, Departament de Comunicació Roc Boronat, 138. 08018 Barcelona, España pere.freixa@upf.edu

\section{Resumen}

La transformación de los medios de comunicación tradicionales en cibermedios supuso, en un primer momento, un proceso de traslación a la red de los formatos y procedimientos definidos para el soporte en papel. Las primeras redacciones digitales integraron los distintos géneros periodísticos, entre los que se encontraban los reportajes especiales de acontecimientos previstos, como son los procesos electorales, las galas o entregas de premios y los festivales musicales. Desde 2005, anualmente, Elpais.com ha publicado de forma continuada reportajes especiales dedicados a los premios Goya y a los premios Oscar. La observación y análisis de esos reportajes interactivos y multimedia permite contemplar no sólo la evolución de los reportajes en los medios digitales sino también la definición y emergencia de un género de reportaje interactivo propio de los cibermedios como son los reportajes planificados por fases.

\section{Palabras clave}

Reportajes planificados por fases; Reportajes especiales; Ciberperiodismo; Reportajes multimedia; Diseño interactivo; Información visual interactiva; Elpais.com; Premios Goya; Oscar Awards.

\begin{abstract}
The transformation of traditional journalism into online journalism has brought with it a process of translating paper-based formats and procedures into online ones. The first digital teams incorporated the different journalistic genres, with special features about planned events such as election processes, galas, awards ceremonies and musical festivals. Since 2005, Elpais.com has continuously published special features devoted to both the Goya and Oscar awards. The observation and analysis of those interactive and multimedia features provides insight not only into the evolution of stories in digital media, but also into the definition and emergence of an interactive genre of digital media such as the story or feature divided into phases.
\end{abstract}

\section{Keywords}

Planed news; Special feature; Online journalism; Cyberjournalism; Multimedia stories; Interaction design; Visual and interactive features; Elpais.com; Premios Goya; Oscar Awards.

Freixa, Pere (2015). "Reportajes especiales en los cibermedios. Análisis de diez años de Premios Goya y Oscar Awards en Elpais.com (2005-2014)”. El profesional de la información, v. 24, n. 3, pp. 291-300.

http://dx.doi.org/10.3145/epi.2015.may.09 


\section{Introducción}

En febrero de 2005 elpais.com realizó por primera vez un interactivo dedicado a los Premios Goya, premios que concede anualmente la Academia de la Artes y las Ciencias Cinematográficas de España. Se trataba de un reportaje hipertextual que se etiquetó en la categoría "especiales" y ocupó durante varios días un recuadro destacado de la portada del medio. Pocas semanas después publicó otro reportaje temático con motivo de la gala de los Oscar de la americana Academy of Motion Picture Arts and Sciences. Desde entonces, cada año y de forma ininterrumpida, elpais.com viene publicando nuevas ediciones especiales de ambos eventos.

A pesar de su distinta dimensión y proyección, los dos acontecimientos despiertan el interés del público. Las retransmisiones televisivas son seguidas por importantes audiencias en ambos casos y provocan el despliegue de las agencias de publicidad para promover y posicionar sus marcas (Lannelongue, 2008; Amorós; Comesaña, 2012).

La primera edición de los dos reportajes en 2005 coincide con un cambio de modelo de negocio de elpais.com. Se abandona el modelo basado exclusivamente en el pago de los contenidos por un modelo mixto, en el que la mayoría de contenidos se ofrecen en abierto y sólo algunos servicios exclusivos se mantienen por suscripción (Salaverría; LópezGarcía; Limia-Fernández, 2005; Salaverría et al., 2005). Ese cambio de política de negocio potenció la publicación de especiales interactivos y multimedia espectaculares, diseñados para todos los públicos y destinados a convertirse en abanderados del nuevo periodismo digital. Para los redactores, creativos y diseñadores multimedia de elpais.com tanto el especial de los Premios Goya como el de los Oscar se convirtieron en un escaparate dónde mostrar sus aptitudes para el diseño interactivo y la innovación.

\section{La comunicación interactiva basa su éxi- to en la capacidad de los autores para diseñar sistemas que organicen los con- tenidos y los comuniquen a los usuarios mediante interfaces a través de un diálo- go interactivo, eficaz y atractivo}

La comunicación interactiva basa su éxito en la capacidad de los autores para diseñar sistemas que organicen los contenidos y los comuniquen a los usuarios mediante interfaces a través de un diálogo interactivo, eficaz y atractivo. En los reportajes especiales de noticias o eventos previsibles, como son las galas de los premios o las campañas electorales, la posibilidad de definir a priori los contenidos permite un grado de planificación y diseño muy superior al que se da habitualmente en una redacción periodística. Dada la semejanza de formato de cada nueva edición con la anterior (mismos apartados, mismas categorías de los premios, largas listas de las fichas de las películas finalistas, fotografías de los premiados, etc.) los autores se ven obligados a innovar cada año en la formalización de los contenidos y en las estrategias de comunicación visual e interactiva.
Diez ediciones después, el análisis comparativo de estos reportajes permite examinar la evolución del medio interactivo en elpais.com y observar la aparición y asentamiento de un género de reportaje interactivo propio de los cibermedios como son los reportajes planificados por fases.

Esta variedad de reportaje, multimedia e interactivo, se distingue por contemplar, en su diseño inicial, una evolución temporal en fases preestablecidas y diferenciadas. En cada fase, el diálogo con los usuarios, la interfaz y la propia estructura de las páginas se adecua al interés específico de la misma. Inconcebible antes de internet y del periodismo digital, elpais.com ha sido capaz de definir y desplegar exitosamente este formato de reportaje en los especiales de los Premios Goya y los Oscar.

La madurez y singularidad de este tipo de reportaje interactivo la corroboran algunos indicadores como, por ejemplo, la incorporación de una categoría específica dedicada a ellos en los prestigiosos Online journalism awards de 2012, la Planned News/Events.

\section{Metodología}

En esta investigación se presentan de forma resumida los resultados del análisis de los 20 interactivos publicados por elpais.com sobre los Premios Goya y Oscar obtenidos mediante la aplicación del sistema de análisis découpage interactivo (Freixa, 2009; Freixa et al., 2014a), el cual permite una observación detallada de los componentes que definen un audiovisual interactivo: estructura, contenidos, interfaces y diálogo interactivo. La aplicación de este protocolo de análisis reporta al investigador un amplio conjunto de indicadores que permiten reestablecer, desde la recepción, el proceso de guión y elaboración del producto analizado.

De ambos interactivos se ha realizado una lectura exhaustiva de la primera y última edición, las de 2005 y 2014, aplicando de forma completa las dos fases del modelo de découpage interactivo, la fase descriptiva y la fase de análisis sistemático. Para las ediciones intermedias (de 2006 a 2013) se ha tomado el análisis de la edición anterior como referente, a partir de la cual se ha realizado una lectura comparativa, localizando los cambios y variantes que se han observado. Dada la repetición de algunos aspectos de toda la serie, como es el tipo de contenidos, la comparación entre ediciones se ha mostrado especialmente significativa con los indicadores descritos en los módulos $d$ y $f$ del modelo de découpage interactivo, referentes a la estructura y a la interfaz (Freixa et al., 2014a).

Se ha mejorado el análisis de la fase descriptiva previsto en el modelo de découpage interactivo incorporado algunos conceptos e indicadores de análisis extraídos del Sistema articulado de análisis de cibermedios, SAAC (Codina et al., 2014) y del modelo de análisis de calidad web (RodríguezMartínez; Codina; Pedraza-Jiménez, 2010).

El estudio se ha realizado utilizando el navegador de un ordenador como dispositivo de lectura. Se han consultado las versiones de los especiales que elpais.com mantiene en activo en su sitio web. Para poder evaluar las variaciones que presenta cada producto durante sus distintas fases (preliminar, acontecimiento, archivo) se ha consultado el regis- 
tro web Wayback Machine de Internet Archive que permite recuperar el estado de una determinada aplicación web en una fecha precisa.

http://archive.org/web

\section{Especiales informativos en los cibermedios. Un género en auge}

La adaptación de los géneros periodísticos tradicionales a la Red y la emergencia de otros nuevos como resultado de la práctica ciberperiodística han sido unos de los principales temas de estudio en los últimos años (Larrondo, 2004; 2009). Los enfoques predominantes se han centrado en las posibilidades hipertextuales, la multimedialidad y la interacción del medio (Aston, 2003; Canavilhas, 2006). Tras unas primeras predicciones marcadamente positivistas y entusiastas, de "utopía tecnológica" en términos de David Domingo (2005), autores como MacGregor (2003) constataron las dificultades para establecer un ámbito informativo interactivo y multimedia: la rápida convergencia tecnológica y empresarial no se estaba trasladando a los contenidos. Se pone de manifiesto entonces la necesidad de situar el foco de atención más allá de la traslación de medios que dicha convergencia estaba propiciando (Masip et al., 2010). Hoy, el estudio de los cibermedios, en tanto que escenario donde se están generando formas autónomas de relato periodístico, ofrece un corpus de trabajos de investigación suficientemente amplio como para reconocer la singularidad, identidad y autonomía de los formatos ciberperiodísticos (Palacios; Díaz-Noci, 2007; Larrondo, 2010; Seixas, 2012; Domínguez, 2013), entre los que destacan formas propias del reportaje digital.

Existe unanimidad a considerar la existencia de reportajes especiales con características propias en el medio digital, aunque se estén empleando distintos términos y matices en su descripción. Para Eva Domínguez (2013, p. 69) “El Especial es un contenido para el que los profesionales del medio han dedicado un esfuerzo creativo, narrativo y tecnológico adicional". Ainara Larrondo (2004) describe dos categorías próximas, el "especial temático" y el "dosier documental". Ambos se caracterizan por incorporar un índice que organiza el contenido y por aprovechar las posibilidades multimedia del medio. Concluye: "se trata de trabajos periodísticos de cariz monográfico, ajenos a la actualidad y referidos a ámbitos especializados" (Larrondo, 2004).

Para cubrir eventos especiales los medios diseñan reportajes en los que muestran su capacidad de innovación y de seducción visual. Estos reportajes temáticos responden a las características descritas por Larrondo: se constituyen como un micro-site autónomo, son independientes del resto de noticias y pueden actualizarse (2010, pp. 190-191).

Sin embargo la comparación de las distintas ediciones que se presenta en este estudio permite observar que algunos especiales temáticos, como son los elaborados por elpais. com en los Goya y los Oscar, responden a unas características específicas que no son comunes a todos los reportajes especiales y que les confieren singularidad. La posibilidad de previsión, tanto de los contenidos como de las fases del evento, determina una estructuración a priori de los mis- mos en fases preestablecidas. Los autores prevén tres momentos diferenciados y correlativos: fase previa, fase de desarrollo del evento y fase de cierre y de archivo. En cada una de ellas no sólo se actualiza la información sino que se modifica la organización jerárquica de las secciones, se reordena la presentación de los contenidos a través de la interfaz y se varían los diálogos con los usuarios. Se trata de reportajes interactivos con varias fases correlativas, a los que hemos llamado reportajes planificados por fases.

\section{Lecturas comparativas a través de la interfaz y la estructura}

Los gráficos 1 y 2 presentan las interfaces diseñadas para las portadas de los reportajes especiales de los Premios Goya y los Oscar durante el período 2005-2014, según se pueden consultar en el archivo de elpais.com e Internet Archive. La comparación permite constatar las continuidades y discontinuidades en el diseño, las adaptaciones a los avances tecnológicos y las tendencias dominantes en la formalización visual del discurso interactivo.

Las tablas 1 y 2 presentan las etiquetas de opciones principales utilizadas en los distintos reportajes durante las diez ediciones analizadas. Se encuentran distribuidas en cuatro áreas diferenciadas de la interfaz: menú superior, menú principal, menú inferior y columna derecha. Para esta comparativa se han descartado las opciones locales y contextuales.

En los tres apartados siguientes se presenta con mayor detalle el resultado del análisis ordenado en tres etapas:

- etapa inicial (2005-08), caracterizada por el uso del texto y del hipertexto;

- etapa intermedia (2009-2012), en la que destaca la incorporación de los usuarios;

- etapa actual (2013-14), definida por el diseño multiplataforma y la planificación en fases.

\section{Primera etapa: texto e hipertexto (2005-2008)}

El análisis de la primera edición del especial de los Goya permite observar los elementos básicos del modelo que se afianzarán y ampliarán en la edición de 2006 y que se corresponden con las observaciones realizadas por Larrondo (2004) al analizar los reportajes especiales en los cibermedios: el especial, definido como una sede autónoma del resto de contenidos del medio, contiene una portada que se constituye como un índice hipertextual desde el que se accede a los distintos contenidos.

\subsection{Contenidos}

En estos dos primeros años se concretan las opciones que organizan los contenidos y que se mantienen prácticamente inalterables durante las diez ediciones: las películas y los premiados son el contenido central del interactivo, siendo el listado de galardonados prácticamente el único material informativo elaborado de forma expresa para el especial. Es interesante constatar como desde la primera edición se aprovechan contenidos realizados previamente. El apartado Películas enlaza con las críticas de los filmes realizadas previamente por los periodistas cinematográficos de elpais. com y publicadas en la sección de Cultura. 


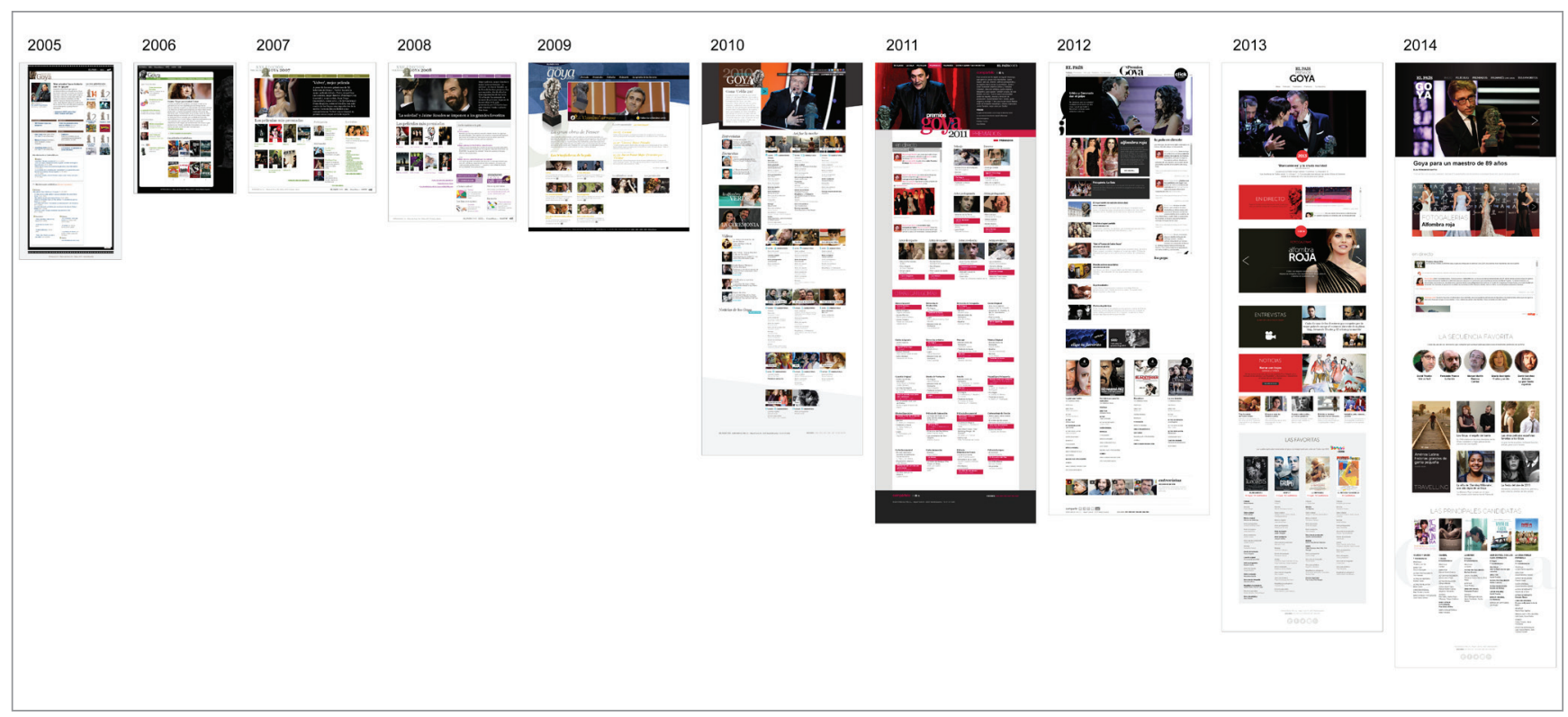

Gráfico 1. Comparación de las portadas de los especiales sobre los Premios Goya en elpais.com (2005-14).

La primera edición del especial de los Goya permite enlazar con una galería fotográfica, entrevistas a los protagonistas y un vídeo de la gala. A partir de la edición de 2006 se incorporan de forma más evidente los materiales promocionales que las distribuidoras y las productoras distribuyen para la promoción de las películas, como son las fichas técnicas, trailers, carteles y fotografías. La primera edición de los Oscar, en 2005, ya incorpora esos materiales promocionales.

\subsection{Patrocinio}

Únicamente en la edición de 2005 de los Goya se publicaron enlaces exclusivos para subscriptores. Es remarcable que esta práctica nunca se realizó en los especiales de los Oscar. A partir del año siguiente todos los contenidos fueron de acceso libre. La tabla 2 permite observar la presencia de enlaces hacia los portales web de los patrocinadores en las tres primeras ediciones de los Oscar: Volvo (2005), Smart (2006), Audi (2007). La falta de patrocinador en la edición de 2008 coincidió con la reducción de complejidad, diseño y planificación del reportaje de aquel año, que volvió a utilizar una portada en forma de índice hipertextual.

Los materiales promocionales que las productoras y distribuidoras proporcionan a los medios se convertirán en parte de los contenidos básicos de ambos productos. En las primeras ediciones de los Oscar condicionarán el diseño de las interfaces.

\subsection{Diseño}

A partir de 2007 se constata el cambio de diseño para adaptar la interfaz a la resolución de 1.024 píxeles de ancho de la pantalla, común en los medios españoles, como acredita Guallar (2007). El cambio de diseño supone también una mayor presencia de los contenidos visuales en la pantalla y la adopción de una gran cabecera fotográfica como característica visual del especial. El diseño de dicha cabecera, formada por una gran fotografía de un protagonista de la gala acompañada por un gran titular, se mantendrá prácticamente inalterable en las ediciones siguientes.
Las ediciones de 2007 y 2008 de los Goya permiten constatar un cambio importante de tendencia en el diseño de aplicaciones interactivas: a pesar de que la portada sigue funcionando como un índice hipertextual, todas las opciones del menú principal presentan los contenidos en un mismo espacio y con un mismo diseño. El desarrollo de la modularidad con el uso de las hojas de estilo CCS2 permite un mayor control de los elementos formales de la pantalla. La hipertextualidad se simula y el usuario percibe una mayor fluidez y unidad del interactivo. Como consecuencia, los especiales ganan unicidad y autonomía respecto del resto de contenidos del medio.

\subsection{Interacción y usuarios}

Encuestas, quinielas y juegos forman parte de las opciones de participación del evento. El especial de los Oscar de 2005 incorpora en el apartado Participe un chat, varios juegos de preguntas y una encuesta. El especial sobre los Goya de ese mismo año únicamente promovió una encuesta en la que se recogían las predicciones de los galardonados. Ambos modelos de interacción parecen responder al deseo de promover un espacio virtual donde los usuarios permanezcan el máximo tiempo posible, combinando información y entretenimiento. Como apunta Ribas (2001) la eficacia en el diseño de un interactivo reside en la capacidad de mantener el interés del usuario a través de la información y el estímulo.

En este primer período elpais.com utiliza el especial como plataforma para fomentar que los usuarios consulten los medios del grupo editorial. Se incorporan etiquetas de navegación en el menú superior o en el inferior que permiten enlazar con las distintas cabeceras del grupo. Esta práctica caerá en desuso en 2007 para los Oscar y en 2008 para los Goya.

\section{Segunda etapa: explotación editorial y usuarios (2009-2012)}

Las ediciones de 2009 evidencian dos cambios sustanciales en la estrategia comunicativa de ambos interactivos: 
Tabla 1. Etiquetas de opciones utilizadas en las 10 ediciones de los Premios Goya por elpais.com, ordenadas según su distribución en la pantalla.

\begin{tabular}{|c|c|c|c|c|c|c|c|c|c|c|c|c|c|c|c|c|c|c|c|c|c|c|c|c|c|c|c|c|c|}
\hline 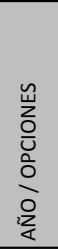 & 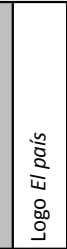 & 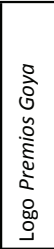 & 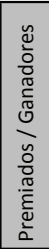 & 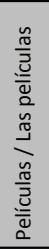 & 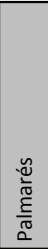 & 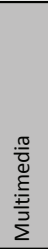 & 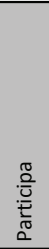 & $\begin{array}{l}\frac{\pi}{0} \\
\frac{\pi}{0} \\
0 \\
0 \\
0 \\
\frac{0}{0} \\
\underline{\underline{U}}\end{array}$ & $\begin{array}{l}\frac{\pi}{\pi} \\
\mathbb{N} \\
\pi\end{array}$ & 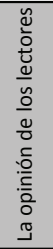 & 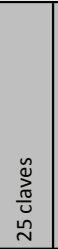 & 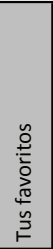 & 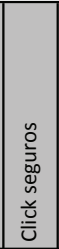 & 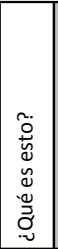 & 荵 & 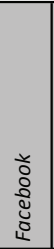 & 离 & हैे & $\begin{array}{l}+ \\
\stackrel{+}{0} \\
\stackrel{0}{\circ}\end{array}$ & $\begin{array}{l}\text { है } \\
\text { ऽँ } \\
\stackrel{\Xi}{\Xi} \\
\Sigma\end{array}$ & $\stackrel{\Xi}{\Xi}$ & $\begin{array}{l}\frac{1}{2} \\
\frac{1}{2}\end{array}$ & $\begin{array}{l}\text { ङे } \\
\text { ठ }\end{array}$ & $\begin{array}{l}\varepsilon \\
\stackrel{0}{\square} \\
\end{array}$ & 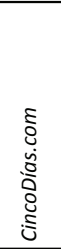 & 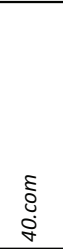 & 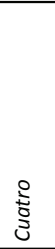 & $\stackrel{m}{\stackrel{m}{u}}$ & 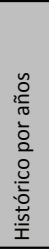 \\
\hline 2005 & A-1 & $\mathrm{P}-1$ & & & & & & & & & & & & & & & & & & & & & & $\mathrm{~A}-2$ & & $A-3$ & & & \\
\hline 2006 & A-1 & $\mathrm{P}-1$ & $\mathrm{P}-2$ & $\mathrm{P}-3$ & $\mathrm{P}-4$ & $\mathrm{P}-5$ & $\mathrm{P}-6$ & & & & & & & & & & & & & & & & & $A-2$ & $\mathrm{~A}-3$ & A-4 & $A-5$ & $A-6$ & \\
\hline 2007 & $\mathrm{E}-1$ & $\mathrm{P}-1$ & $\mathrm{P}-2$ & P-3 & $\mathrm{P}-4$ & $\mathrm{P}-6$ & $\mathrm{P}-7$ & & $\mathrm{P}-5$ & & & & & & & & & & & & & & & $\mathrm{E}-2$ & $\mathrm{E}-3$ & $\mathrm{E}-5$ & $E-4$ & & \\
\hline 2008 & $\mathrm{E}-1$ & $\mathrm{P}-1$ & $\mathrm{P}-2$ & $\mathrm{P}-3$ & $\mathrm{P}-4$ & $\mathrm{P}-6$ & $\mathrm{P}-7$ & & P-5 & & & & & & & & & & & & & & & $\mathrm{E}-2$ & $\mathrm{E}-3$ & \begin{tabular}{|l|l|}
$E-5$ \\
\end{tabular} & $E-4$ & & \\
\hline 2009 & $\mathrm{~A}-1$ & P-1 & $\mathrm{P}-3$ & $\mathrm{P}-4$ & $\mathrm{P}-5$ & & & $\mathrm{P}-2$ & & $\mathrm{P}-6$ & & & & & & & & & & & & & & & & & & & $\mathrm{E}-1$ \\
\hline 2010 & $\mathrm{P}-2$ & P-1 & $\mathrm{P}-4$ & $\mathrm{P}-5$ & $\mathrm{P}-6$ & & & P-3 & & $\mathrm{P}-7$ & & & & A-1 & & $\mathrm{A}-2$ & $\mathrm{~A}-3$ & & & A-4 & $\mathrm{A}-5$ & A-6 & \begin{tabular}{|l|}
$A-7$ \\
\end{tabular} & & & & & & $\mathrm{E}-1$ \\
\hline 2011 & $P-7$ & $n / a$ & $\mathrm{P}-4$ & P-3 & $P-5$ & & & & $\mathrm{P}-2$ & & $\mathrm{P}-1$ & $P-6$ & & & A-1 & $\mathrm{A}-2$ & $A-3$ & & & & & & & & & & & & \\
\hline & & & & & & & & & & & & & & & $\mathrm{E}-1$ & $\mathrm{E}-2$ & $\mathrm{E}-3$ & & & & & & & & & & & & $\mathrm{E}-4$ \\
\hline 2012 & $\mathrm{~A}-1$ & $\mathrm{P}-6$ & $\mathrm{P}-2$ & $\mathrm{P}-3$ & $\mathrm{P}-4$ & & & $\mathrm{P}-1$ & & & & P-5 & $\mathrm{P}-7$ & & $\mathrm{E}-1$ & $\mathrm{E}-2$ & $\mathrm{E}-3$ & $E-4$ & $\mathrm{E}-5$ & & & & & & & & & & $E-6$ \\
\hline 2013 & $\mathrm{~A}-1$ & $A-2$ & $\mathrm{P}-3$ & $\mathrm{P}-2$ & $\mathrm{P}-4$ & & & $\mathrm{P}-1$ & & & & $\mathrm{P}-5$ & & & $\mathrm{E}-1$ & $\mathrm{E}-2$ & $E-3$ & $E-4$ & $\mathrm{E}-5$ & & & & & & & & & & $\mathrm{E}-6$ \\
\hline 2014 & $\mathrm{~A}-1$ & $A-2$ & $\mathrm{P}-3$ & $\mathrm{P}-2$ & $\mathrm{P}-4$ & & & P-1 & & & & $\mathrm{P}-5$ & & & $\mathrm{E}-1$ & $\mathrm{E}-2$ & $\mathrm{E}-3$ & $\mathrm{E}-4$ & $\mathrm{E}-5$ & & & & & & & & & & $E-6$ \\
\hline \multicolumn{6}{|c|}{ Menú superior (S) } & & & & & & & & & & & & & & & & & & & & & & & & \\
\hline \multicolumn{6}{|c|}{ Menú principal $(P)$} & & & & & & & & & & & & & & & & & & & & & & & & \\
\hline \multicolumn{6}{|c|}{$\begin{array}{l}\text { Zona de contenidos (C) } \\
\text { [n/a = opción inactiva] }\end{array}$} & & & & & & & & & & & & & & & & & & & & & & & & \\
\hline \multicolumn{6}{|c|}{ Menú inferior (I) } & & & & & & & & & & & & & & & & & & & & & & & & \\
\hline
\end{tabular}

- el primero consiste en la eliminación de las etiquetas de los otros medios del grupo editorial. El acceso a las distintas cabeceras se sustituye por el acceso a las ediciones anteriores del especial. Se apuesta por una navegación que promueve la consulta y explotación del propio archivo de especiales interactivos.

- el segundo radica en incorporar en el menú principal la opción La quiniela de los lectores en el especial de los Oscar y La opinión de los lectores en el de los Premios Goya. Ambas decisiones suponen el inicio de un giro editorial que pretende dar respuesta a los intereses y opiniones de los lectores, en detrimento de la promoción corporativa.

\subsection{Ensayos de prueba y error}

El diseño de los especiales de los Oscar durante este período presenta un nivel de variabilidad mucho mayor que en los Premios Goya. Las tablas 1 y 2 muestran la poca continuidad en la definición de las estructuras de navegación y etiquetado en ambos productos. Los menús principales incorporan nuevas etiquetas que entran en desuso al año siguiente y que son sustituidas por otras que tampoco persisten. El diseño de una buena arquitectura de la información (Pérez-Montoro, 2010) se convierte en ese período en una de las principales tareas de los diseñadores de interactivos. El rediseño de cada especial se acompaña con una revisión completa de opciones de navegación, localización en pantalla y tratamiento del contenido.

\subsection{Irrupción de las redes sociales}

El especial de los Premios Goya incorpora etiquetas de enlace a las redes sociales en 2010. El especial de los Oscar lo hará el año siguiente. La presencia de las redes sociales en los especiales permite tanto la promoción del contenido a través internet como el diálogo con los usuarios. Este cambio parece ser un indicador de la tendencia observada en otros cibermedios por Rodríguez-Martínez, Codina y Pedraza (2010).

El menú de los Goya de esa edición incorpora la opción de ayuda ¿Qué es esto? que da acceso a un texto en el que se muestran los objetivos de esa nueva estrategia: "Todos los iconos apuntan a servicios web externos y ajenos a elpais. com que facilitan la gestión personal o comunitaria de la información. Estos servicios permiten al usuario, por ejemplo, clasificar, compartir, valorar, comentar o conservar los contenidos que encuentra en internet".

En la edición del especial de los Oscar de 2010 y en la de los Goya de 2011 la participación de los usuarios es recompensada con sorteos de entradas en la página de elpais.com en Facebook.

\subsection{Integración de medios y estrategia transmedia}

Los diseños de las portadas de este período se transforman: dejan de plantearse como índices hipertextuales para convertirse en escaparates que integran e incluyen los contenidos multimedia. La cabecera del especial de los Premios Goya 2010, por ejemplo, incorpora una adaptación en formato carrusel de la galería fotográfica sin necesidad de cambiar de página. Esta herramienta también se utilizará en 2011 en el especial de los Oscar.

En la portada de los Oscar 2009 por primera vez se activan dos vídeos de la gala en el centro de la pantalla sin necesidad de dar ningún salto hipertextual. Este recurso se mantiene en las ediciones posteriores. De este modo los contenidos empiezan a ser accesibles desde la portada y se consigue eliminar los enlaces textuales que son sustituidos por los mismos contenidos. 


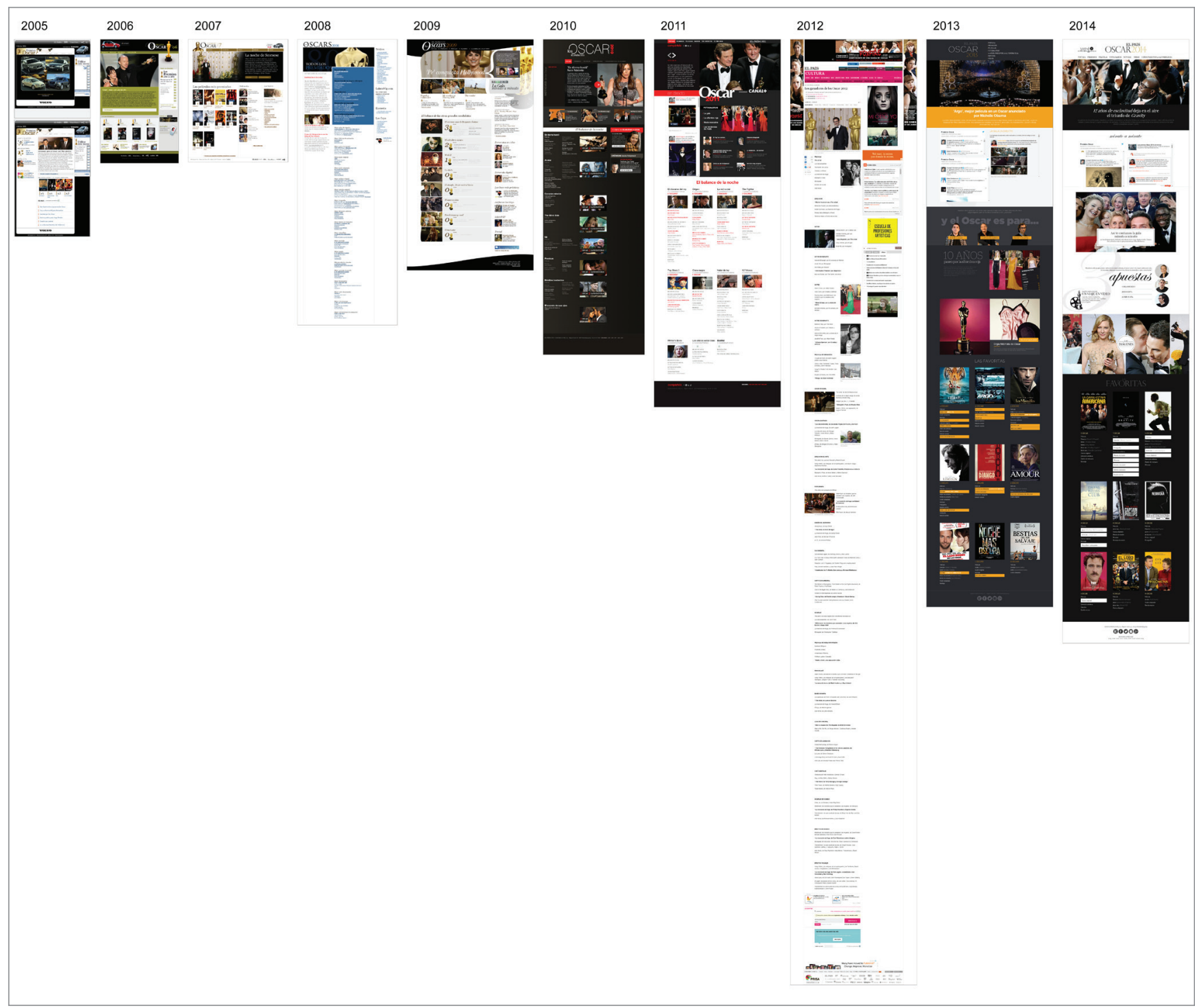

Gráfico 2. Comparación de las portadas de los especiales sobre los Oscar en elpais.com (2005-14).

En los especiales de 2011 de los Goya y los Oscar se incorporan las publicaciones que los redactores realizan en directo durante la gala. La agregación de los posts publicados en la red social Eskup se visualiza desde de la portada del especial. La visualización de las redes sociales en la interfaz del interactivo persigue una doble función:

- añade actualización e inmediatez al interactivo, uno de los aspectos más valorados por los usuarios;

- convierte el interactivo en contenedor de archivo para facilitar el posterior acceso a los contenidos generados en la red social.

\section{Tercera etapa: multiplataforma y planificación por fases (2013-2014)}

Las ediciones de 2013 y 2014 de ambos premios presentan prácticamente la misma arquitectura de información por lo que respecta a la distribución de los tres menús analizados. Sin embargo la distribución en pantalla y formalización entre unas y otras ediciones varía sustancialmente. Cada uno de los reportajes supone una importante producción audiovisual e interactiva: la edición de 2014 de los Oscar, por ejemplo, contempla 9 interfaces. Dos de ellas, Paseo por la alfombra roja y Vota el Oscar de los Oscar pueden considerarse recursos interactivos con guión y funciones propias. El reportaje contiene más de 300 fotografías y 4 vídeos además de los trailers, fichas técnicas y otros contenidos publicitarios aportados por las distribuidoras de cada una de las películas finalistas. Los contenidos se complementan con las aportaciones de los críticos cinematográficos del medio que valoran cada una de las películas.

\subsection{Multiplataforma}

A partir de 2013 el diseño de los especiales contempla la diversidad de dispositivos utilizados por los usuarios para acceder a la información. La necesidad de potenciar y facilitar la accesibilidad a la información (Díaz-Noci, 2010) propicia el uso de recursos de diseño web adaptable (responsive design). La combinación del nuevo lenguaje de programación HTML5, las posibilidades de las hojas de estilo CSS3 y la utilización de recursos de Javascript menos obstructivos permiten a los autores imaginar interfaces capaces de adaptarse de forma aceptable a los distintos dispositivos de consulta que están utilizando los usuarios: ordenadores, tabletas y teléfonos inteligentes. 
Tabla 2. Etiquetas de opciones utilizadas en las 10 ediciones de los premios Oscar por elpais.com, ordenadas según su distribución en la pantalla.
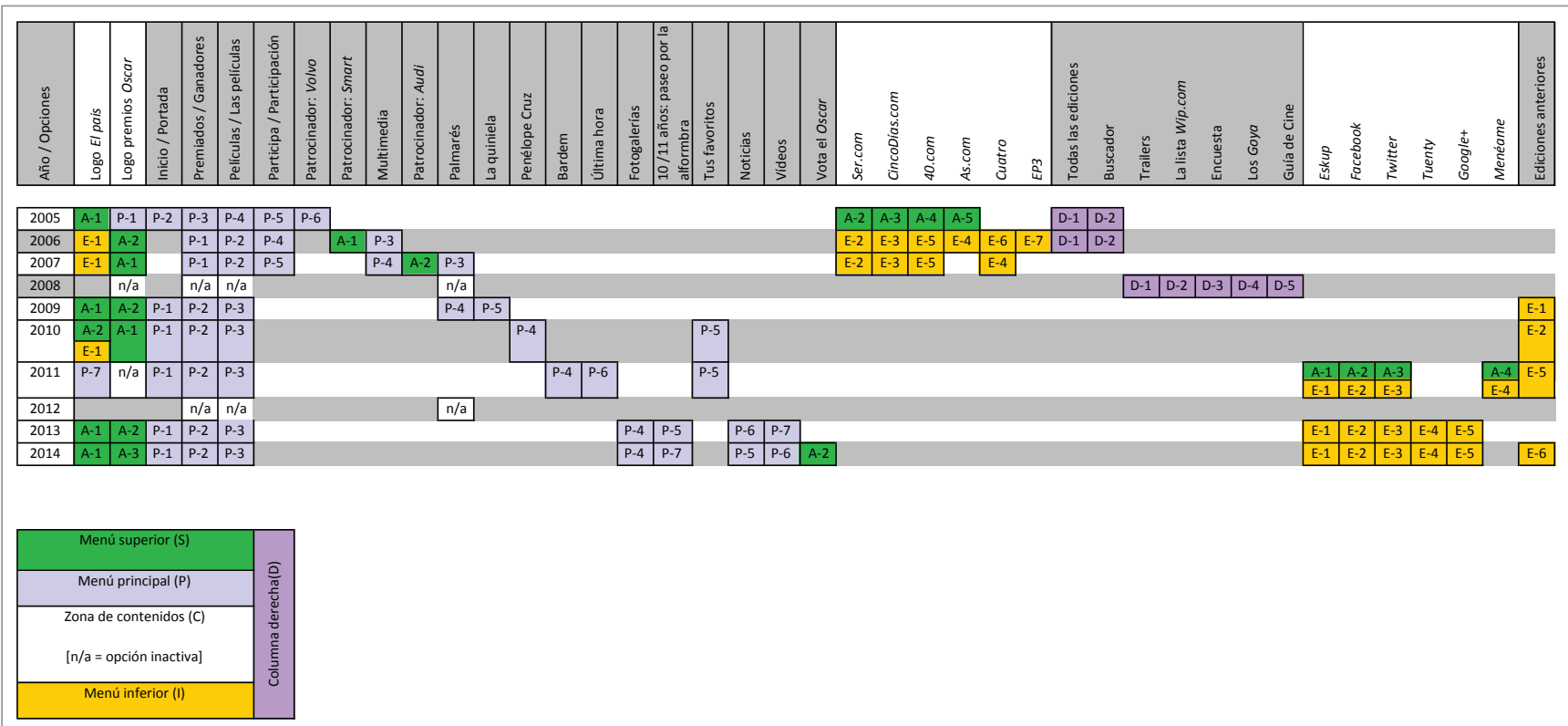

Uno de los efectos reconocibles del uso de los recursos del diseño web adaptable es la reducción de columnas en la organización de la pantalla. El formato en columnas, propio del diseño periodístico en papel, es substituido por el diseño en bloques agrupados en una columna única. Este cambio provoca documentos de una gran extensión, como se puede comprobar en los gráficos 1 y 2 . La portada del reportaje de los Oscar de 2014 es cuatro veces mayor que la de 2005.

A partir de 2013 el diseño de los especiales contempla la diversidad de dispositivos utilizados por los usuarios para acceder a la información

El diseño multiplataforma incrementa la integración de los contenidos multimedia iniciada años antes. Para facilitar la lectura desde distintos dispositivos se utilizan visualizaciones en las que se eliminan los marcos que delimitan los bloques, consiguiendo con ello una gran sensación de transparencia. La activación de una galería o la puesta en marcha de un vídeo se realiza sin, aparentemente, activar ningún plug-in u otro programa complementario, simplificando enormemente la interacción con los contenidos y dando lugar a una mayor continuidad visual y dramática. La integración de medios está siendo uno de los elementos de mayor calado en la transformación de los reportajes interactivos en los cibermedios y uno de los elementos que parece estar transformando los hábitos de lectura de los lectores digitales (Freixa et al., 2014b).

\subsection{Planificación por fases}

La complejidad de los especiales actuales se debe a la cantidad de contenido que incorporan y al número de documentos e interfaces que se deben idear y crear. Suponen además un reto de comunicación y diálogo con los usuarios. El éxito de un especial ya no se mide por el número de accesos que pueda contabilizar durante el evento relatado sino por su capacidad de incidir en la audiencia durante un determinado período de tiempo, que puede ser de días o semanas según el tipo de acontecimiento que se noticia. La captura en días sucesivos de la portada del interactivo de los Premios Goya de 2014 (gráfico 3) permite constatar la planificación del mismo en tres fases: preliminar, desarrollo y final y de archivo.

\section{El formato en columnas es substituido por el diseño en bloques agrupados en una columna única. Este cambio provoca documentos de una gran extensión}

\section{a) Fase preliminar}

Durante la fase preliminar, que se inició nueve días antes de la gala de entrega de los premios, se publicó una primera versión del especial con el anuncio del concurso El Goya de los Goya. Consistía en una votación popular para elegir la mejor película entre las premiadas en las 27 ediciones anteriores del certamen. Dos días antes, el 31 de enero, se publicitó el concurso en las páginas de cultura de elpais.com. Como se puede apreciar en el gráfico 3, tanto la edición del periódico digital como la aplicación interactiva se hicieron eco de los avances de la votación de los lectores, ofreciendo noticias relacionadas e incorporando el resultado de las mismas en la portada del especial. El día 7 de febrero se publicó un amplio reportaje de la película Todo sobre mi madre, el filme elegido por los usuarios como mejor Goya de todas las ediciones. La captura de la portada del especial realizada el 9 de febrero incorpora el resultado de la votación y ya no cuenta con el anuncio del concurso, finalizado. 


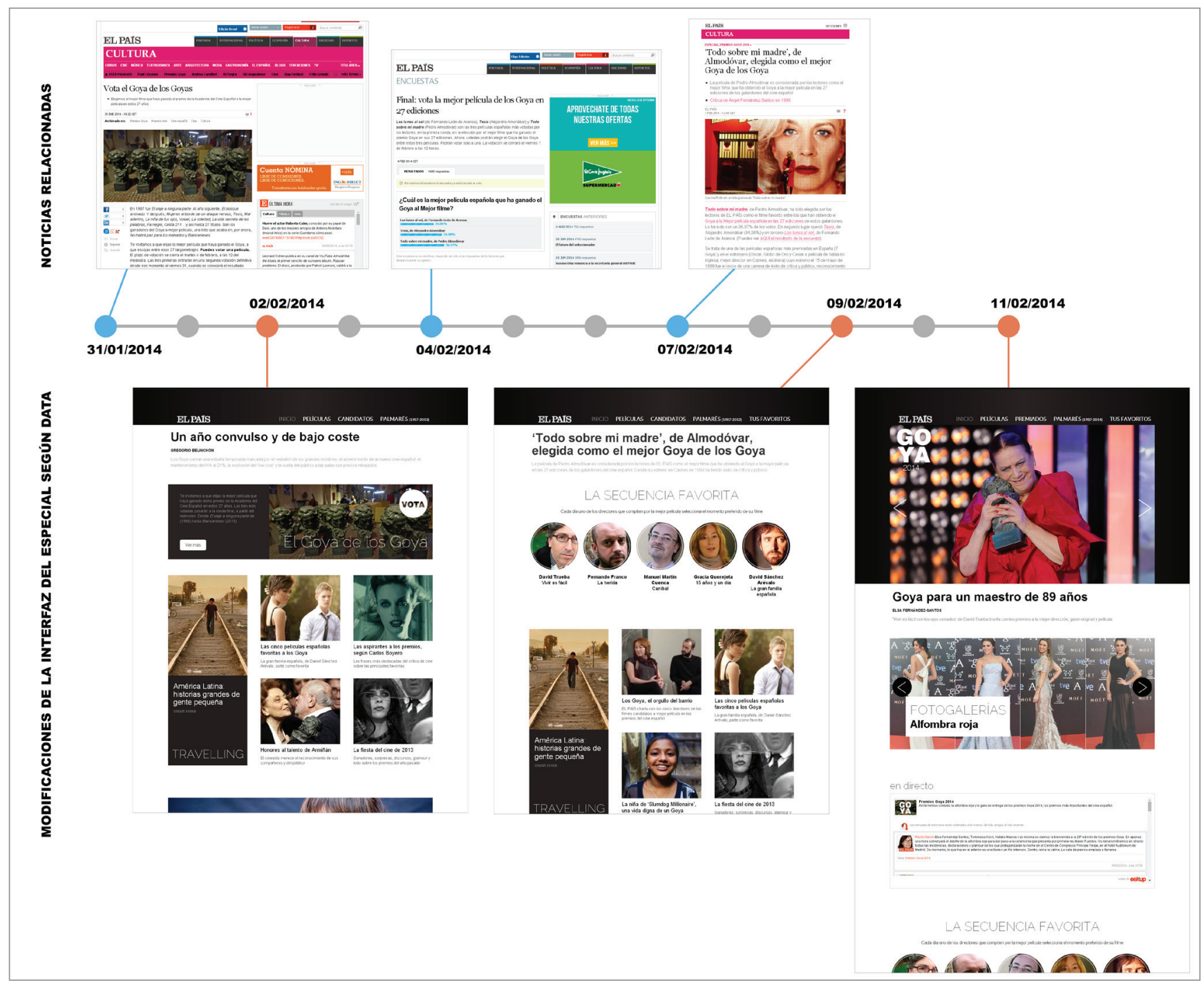

Gráfico 3. Composición en la que se pueden apreciar tres actualizaciones de la portada del especial de los Premios Goya 2014 así como capturas de las noticias publicadas vinculadas al evento en elpais.com.

\section{b) Fase de desarrollo}

Durante la fase de desarrollo, mientras acontece la gala, la portada principal sustituye los bloques del concurso e incorpora un módulo (widget) que permite seguir los posts que los redactores de elpais.com están publicando en el blog creado para el evento en eskup.elpais.com. Los reporteros y periodistas desplegados insertan breves comentarios, fotografías o enlaces a otras informaciones que están aconteciendo en las redes sociales.

El éxito de un especial ya no se mide por el número de accesos sino por su capacidad de incidir en la audiencia durante un determinado período de tiempo

En esta fase se promueve la participación de los espectadores con un concurso en el que se busca adivinar quiénes serán finalmente los galardonados y en el que se sortean entradas para asistir gratis al cine.

\section{c) Fase final y de archivo}

En la fase final y de archivo se añaden todos los contenidos y se activan todos los enlaces del reportaje interactivo. Se editan todas las páginas con las informaciones derivadas de la gala. El especial concluido se publicita en la portada de elpais.com y se incorpora al archivo de "especiales" para su consulta posterior.

\section{Conclusiones}

El análisis de las 10 ediciones de especiales de los Premios Goya y Oscar realizados por elpais.com permite constatar la transformación de los reportajes especiales en los cibermedios. Han dejado de ser una compilación de recursos indexados para convertirse en complejas estructuras informativas interactivas y multimedia que se planifican y evolucionan durante un determinado período de tiempo. Estos nuevos reportajes, que hemos nombrado como reportajes planificados por fases, contemplan una estrategia comunicacional global. El diseño del propio interactivo, los redactores y comunicadores implicados así como los contenidos y actividades destinados a los usuarios varían en cada fase, adaptándose y creando un relato de los acontecimientos acorde con 
la experiencia compartida con los usuarios. De esta forma el interactivo se convierte no sólo en un espacio en el que se comparte la información sino que, mediante la implicación del público y de las redes sociales, restituye la experiencia del evento noticiado. En cada nueva edición, el reto de la comunicación interactiva: facilitar el acceso a los contenidos a través de la interfaz y seducir al usuario, obliga a los autores a imaginar recursos que renueven el interés del público.

Los especiales han dejado de ser una compilación de recursos indexados para convertirse en complejas estructuras informativas interactivas y multimedia que se planifican y evolucionan durante un determinado período de tiempo

\section{Notas}

1. Este trabajo forma parte del proyecto Audiencias activas y periodismo. Interactividad, integración en la web y buscabilidad de la información periodística. CSO201239518-C04-02. Plan Nacional de I+D+i, Ministerio de Economía y Competitividad, España.

2. Webs consultadas

- Primera edición del especial sobre los Premios Goya en elpais.com http://www.elpais.com/especial/premios-goya/2005

- Acceso a todos los especiales sobre los Premios Goya en elpais.com

http://elpais.com/especiales/premios-goya

- Primera edición del especial sobre los Oscar en elpais.com http://www.elpais.com/especial/oscars/2005

- Acceso a todos los especiales sobre los Oscar en elpais.com http://elpais.com/especiales/oscars

- Página de la Online News Association dedicada a los Online Jounalism Awards

http://journalists.org/awards

- Página principal de acceso a la Wayback Machine, de Internet Archive:

https://archive.org/web

- Página de la ayuda ¿Qué es esto? en elpais.com: http://www.elpais.com/QueEsEsto.html

\section{Bibliografía}

Amorós, Anna; Comesaña, Patricia (2012). "Las relaciones públicas como estrategia de comunicación en los eventos cinematográficos: los Premios Goya". Revista internacional de relaciones públicas, v. 2, n. 3, p. 113-130.

http://dx.doi.org/10.5783/RIRP-3-2012-06-113-130

Aston, Judith (2003). Interactive multimedia: an investigation into its potential for communicating ideas and arguments. Tesis doctoral. Londres: Royal College of Art.

Canavilhas, João (2006). Web journalism: from the inverted pyramid to the tumbled pyramid.

http://www.bocc.uff.br/pag/canavilhas-joao-invertedpyramid.pdf
Codina, Lluís; Pedraza, Rafael; Díaz-Noci, Javier; RodríguezMartínez, Ruth; Pérez-Montoro, Mario; Cavaller-Reyes, Víctor (2014). "Sistema articulado de análisis de cibermedios (SAAC): una propuesta sobre el qué y el cómo para estudiar medios de comunicación digitales". Hipertext.net, n. 12.

http://raco.cat/index.php/Hipertext/article/view/275560/364530

Díaz-Noci, Javier (2010). “Medios de comunicación en internet: algunas tendencias". El profesional de la información, v. 19, n. 6, pp. 561-567.

http://dx.doi.org/10.3145/epi.2010.nov.01

Domingo, David (2005). "The difficult shift from utopia to realism in the internet era. A decade of online journalism research: theories, methodologies, results and challenges". En: $1^{\text {st }}$ European communication conf., Amsterdam: ECCR, ECA.

http://www.makingonlinenews.net/docs/domingo_ amsterdam2005.pdf

Domínguez, Eva (2013). El periodismo inmersivo: fundamentos para una forma periodística basada en la interfaz y la interacción. Tesis doctoral. Barcelona: Universitat Ramon Llull.

http://www.tdx.cat/handle/10803/108956

Freixa, Pere (2009). Modelo para el découpage de audiovisuales interactivos. Universitat Pompeu Fabra. http://hdl.handle.net/10230/16197

Freixa, Pere; Soler-Adillon, Joan; Sora, Carles; Ribas, J. Ignasi (2014a). "Aportaciones del découpage interactivo en la lectura y análisis de audiovisuales interactivos de los cibermedios". Hipertext.net, n. 12.

http://raco.cat/index.php/Hipertext/article/view/274410/364411

Freixa, Pere; Soler-Adillon, Joan; Sora, Carles; Ribas, J.Ignasi (2014b). "Snow fall y A short history of the highrise: dos modelos de comunicación audiovisual interactiva del New York Times". En: XX Congreso intl de la Sociedad Española de Periodística. Barcelona, 2014.

http://gci.upf.edu/node/112

Guallar, Javier (2007). "La renovación de los diarios digitales: rediseños y web 2.0". El profesional de la información, v. 16, n. 3, pp. 235-242.

http://www.elprofesionaldelainformacion.com/contenidos/2007/ mayo/08.pdf http://dx.doi.org/10.3145/epi.2007.may.08

Lannelongue, Marie-Pierre (2008). Los secretos de la moda al descubierto. Barcelona: Gustavo Gili. ISBN: 978 8425222115

Larrondo, Ainara (2004). "El reportaje se reinventa en la red: estructura del reportaje hipertextual". Revista latina de comunicación social, n. 57, enero-junio 2004.

http://www.ull.es/publicaciones/latina/20040357larrondo. htm

Larrondo, Ainara (2009). "La metamorfosis del reportaje en el ciberperiodismo: concepto y caracterización de un nuevo modelo narrativo". Comunicación y sociedad, v. 22, n. 2, pp. 59-88.

http://www.unav.es/fcom/communication-society/es/ articulo.php?art_id=317 
Larrondo, Ainara (2010). "The potential of web-only feature stories. A case study of Spanish media sites". Journalism studies, v. 12, n. 2, pp. 188-204.

http://dx.doi.org/10.1080/1461670X.2010.491300

MacGregor, Phil (2003). "Mind the gap: Problems of multimedia journalism". Convergence, v. 9, n. 8, pp. 8-17. http://dx.doi.org/10.1177/135485650300900302

Masip, Pere; Díaz-Noci, Javier; Domingo, David; Micó-Sanz, Josep-Lluís; Salaverría, Ramón (2010). “Investigación internacional sobre ciberperiodismo: hipertexto, interactividad, multimedia y convergencia". El profesional de la información, v. 19, n. 6, pp. 568-576.

http://www.elprofesionaldelainformacion.com/contenidos/2010/ noviembre/02.pdf

http://dx.doi.org/10.3145/epi.2010.nov.02

Palacios, Marcos; Díaz-Noci, Javier (eds.) (2007). On-line journalism: research methods. A multidisciplinary approach in comparative perspective. Bilbao: Universidad del País Vasco. ISBN: 9788498601916

Pérez-Montoro, Mario (2010), "Arquitectura de la información en entornos web". El profesional de la información, v. 19, n. 4, pp. 333-337.

http://www.elprofesionaldelainformacion.com/contenidos/2010/ julio/01.pdf

http://dx.doi.org/10.3145/epi.2010.jul.01

Ribas, Joan-Ignasi (2001). "Difusió cultural i comunicació au- diovisual interactiva". Temes de disseny, n. 18, pp. 170-204. http://tdd.elisava.net/coleccion/18/ribas

Rodríguez-Martínez, Ruth; Codina, Lluís; Pedraza-Jiménez, Rafael (2010). "Cibermedios y web 2.0: modelo de análisis y resultados de aplicación". El profesional de la información, v. 19, n. 1 , pp. $35-44$

http://www.elprofesionaldelainformacion.com/contenidos/2010/ enero/05.pdf

http://dx.doi.org/10.3145/epi.2010.ene.05

Salaverría, Ramón; Díaz-Noci, Javier; López-García, Xosé; Palomo, María-Bella (2005). "Spain: multimedia richness and variety of business models". En: Van-der-Wurff, Richard; Lauf, Edmund (eds.). Print and online newspapers in Europe. A comparative content analysis in 16 countries in Western and Eastern Europe. Amsterdam: Het Spinhuis Publishers, pp. 231- 243. ISBN: 9789055892389.

Salaverría, Ramón; López-García, Xosé; Limia-Fernández, Moisés (2005). "Análisis comparativo de cibermedios: Lavanguardia.es, Elmundo.es y Elpaís.es". En: Salaverria, Ramon (ed.). Cibermedios. El impacto de internet en los medios de comunicación en España, Sevilla: Comunicación Social, pp. 125-143. ISBN: 9788496082335.

Seixas, Lia (2012). "Género periodístico: combinaciones y regularidades en sitios informativos". En: Cabrera, MaríaÁngeles (cord.). Evolución de los cibermedios. De la convergencia digital a la distribución multiplataforma. Madrid: Fragua, pp. 301-318. ISBN: 9788470745669.

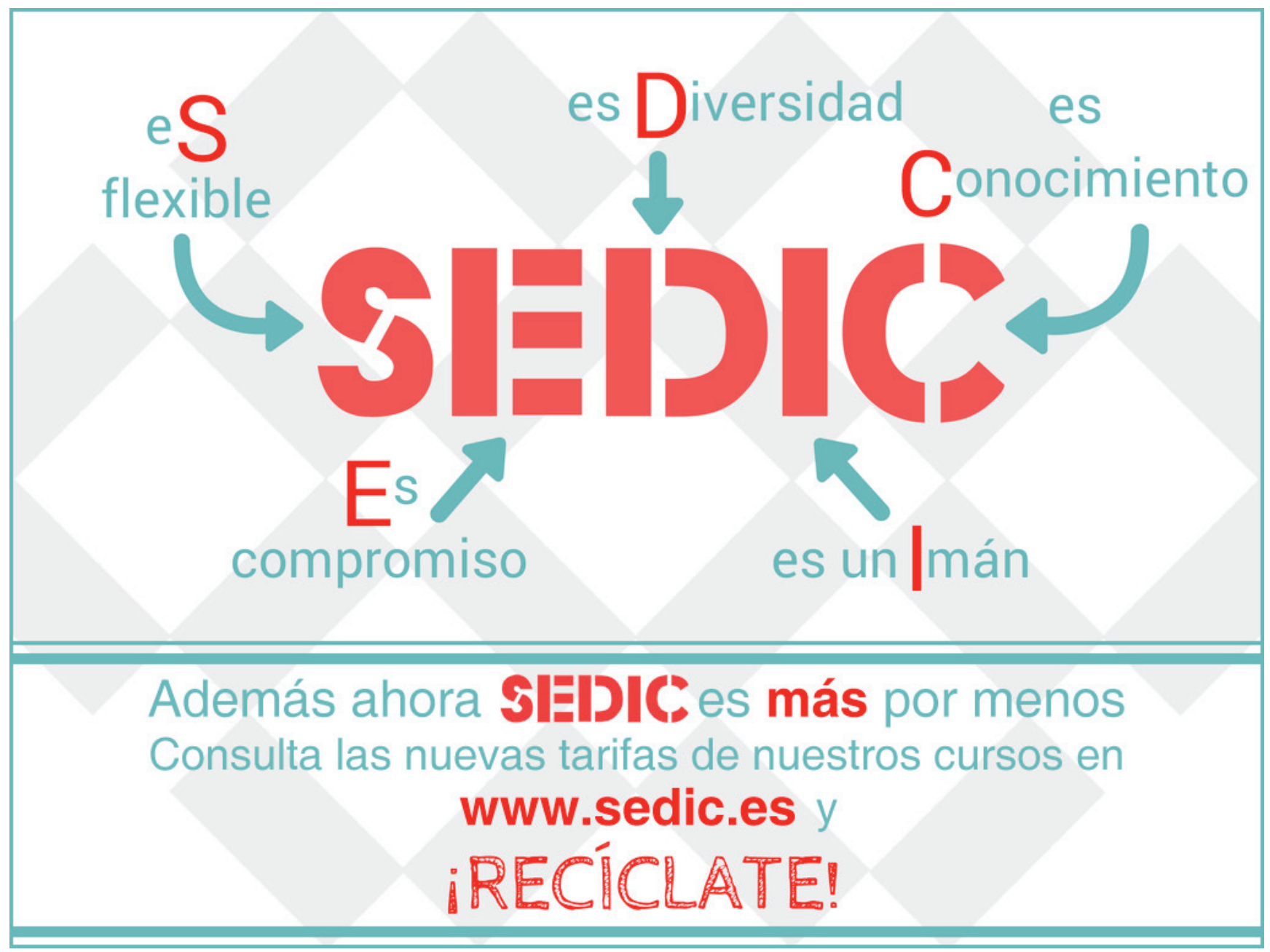

